\title{
Context effects in repetition priming are sense effects
}

\author{
J. VIVIEN BAINBRIDGE and STEPHAN LEWANDOWSKY \\ University of Oklahoma, Norman, Oklahoma \\ and \\ KIM KIRSNER \\ University of Western Australia, Nedlands, Western Australia, Australia
}

\begin{abstract}
This article reports three experiments that investigate the role of context in repetition priming using a lexical decision task. The experiments show that repetition priming is either eliminated or significantly reduced if a change in context also alters the perceived sense of a nonhomographic target word. If perceived sense is not altered, a change in context is inconsequential. This points to the important role played by perceived sense in repetition priming. An explanation within a sense-specific activation framework is proposed in preference to a modified processing view.
\end{abstract}

Repeated processing of a stimulus often facilitates performance on a cognitive task. This facilitation is known as repetition priming and, because it can arise in the absence of conscious recollection of the original stimulus presentation, is often assumed to reveal an implicit form of memory. Repetition priming is a very reliable phenomenon and has been observed in numerous tasks-for example, lexical decision (see, e.g., Scarborough, Cortese, \& Scarborough, 1977) and word-fragment completion (see, e.g., Tulving, Schacter, \& Stark, 1982). This article reports three repetition priming experiments, using lexical decision, in which the effect of linguistic context on performance was investigated. Our investigation was motivated by two concerns: first, to contribute to the reshaping of the prevailing theoretical views of implicit memory and, second, to explore possible reasons for the diversity of previously reported context effects.

Experimental and theoretical activity has focused on implicit memory for little over a decade. During this period, the prevailing theoretical approaches have become increasingly complex, gradually shifting from presumed low-level perceptual phenomena (e.g., perceptual fluency; Jacoby \& Dallas, 1981) to include higher-level cognitive processes (e.g., levels of processing; Hamann, 1990). This trend is exemplified by the growing interest in the effects of a change in linguistic context between study and test. Context here refers to encoding cues that are presented with the stimulus-in the pair SMOOTH-FILE, for example, SMOOTH provides the context for the target item

\footnotetext{
This research was supported in part by a grant from the Australian Research Grants Scheme to the third author. The authors gratefully acknowledge comments on earlier drafts of this article from Francis $T$ Durso, Scott D. Gronlund, William E. Hockley, Colin M. MacLeod, Arthur Samuel, and several anonymous reviewers. Correspondence concerning this article should be addressed to J. Vivien Bainbridge, Department of Psychology, University of Oklahoma, Norman, OK 73019-0535.
}

FILE. Whereas an earlier review by Monsell (1985) cited only one study on context effects in repetition priming (Carroll \& Kirsner, 1982), today's list includes numerous experiments in a variety of paradigms. The catalogue of empirical findings has thus been extended considerably, but the observed effects, often tied to a specific method (e.g., stem completion; Graf \& Schacter, 1987) or class of stimuli (e.g., homographs; Masson \& Freedman, 1990), have so far failed to form a fully coherent picture.

We find it helpful to present previous results according to the degree of stimulus reconstruction required by the memory test. Some tasks, such as lexical decision tasks, involve presentation of an intact stimulus, whereas others, such as stem completion tasks, present only part of the stimulus and require reconstruction of the target. The former tasks are said to require only data-driven processing, whereas the latter are said to require more conceptually driven processing, because some task-relevant information is missing. The distinction between data-driven and conceptually driven processes underlies the influential processing view of implicit memory (see, e.g., Kolers \& Roediger, 1984; Roediger \& Blaxton, 1987) and may also speak to the likely context sensitivity of various tasks: Because it is extraneous to the stimulus, linguistic context should be expected to have a greater effect on conceptually driven reconstruction than on data-driven decisions about an intact stimulus.

Indeed, primed stem completion has repeatedly been shown to be sensitive to a shift in context between study and test (see, e.g., Graf \& Schacter, 1985, 1987, 1989). When subjects are instructed to complete the stem wIN_ with the first word that comes to mind, the probability of giving a particular response (e.g., WINDOW) from the set of possible completions (e.g., WINDOw, WINNER, WINE) is elevated by prior study. Completion probabilities are further elevated when context cues present at study and test are identical. 
Experiments using lexical decision or other more datadriven tasks, in contrast, have produced a more diverse pattern. A useful taxonomy of these studies distinguishes between the different ways in which context was manipulated: Some experiments provided context at study, but not at test, and reported no differential effects of context (see, e.g., Jacoby, 1983), whereas others used single words to manipulate context and obtained rather mixed outcomes (see, e.g., Carroll \& Kirsner, 1982; Masson $\&$ Freedman, 1990). We argue that there may be methodological reasons for preferring the latter class of studies and that the remaining empirical discrepancies can be resolved by using a more extensive and concise manipulation of context.

Consider experiments in which context was provided at study but not at test (see, e.g., Allen \& Jacoby, 1990; Blaxton, 1989; Jacoby, 1983; Levy \& Kirsner, 1989; MacLeod, 1989; Oliphant, 1983). When single words formed the context manipulation, as in Jacoby's (1983) experiment, study items were accompanied by various cues-for example, an antonym (HOT-COLD) or a related item (SNOw-COLD) versus a neutral letter pattern (XXX-COLD). At a later test, with words in isolation (COLD), less priming was observed for the antonym and related conditions than for the neutral condition. Similarly, when study items were part of a meaningful text, priming on a later isolated presentation was minimal (MacLeod, 1989) or absent (Levy \& Kirsner, 1989; Oliphant, 1983). However, rather than concluding on the basis of these data that study context disrupts implicit memory for individual words, we argue that the foregoing studies may have failed to find beneficial effects of context by not re-presenting it at test. In Jacoby's experiment, for example, there is no way of knowing whether reprocessing COLD at test, in the presence of SNOW, might not have led to greater facilitation than was observed when COLD was tested in isolation.

Turning to studies that provided contextual cues at study and test, Carroll and Kirsner (1982) defined context as the co-occurrence of two words in a pair and showed that repetition of context can facilitate lexical decision over and above mere repetition of the stimulus words, provided that the items in repeated pairs were preexperimentally associated. When the items in a pair were not preexperimentally related, in contrast, repetition of the intact pair provided no more priming than when the items were rearranged across different pairs. More recently, Smith, MacLeod, Bain, and Hoppe (1989) reported two experiments using unrelated pairs in which repetition priming, albeit across short retention intervals, was equal for both intact and rearranged pairs. Taken together, the studies show that when context is provided by a single cue word, and when the context-target pairs are preexperimentally unrelated, manipulations of context appear to have inconsequential effects.

In contrast, Masson and Freedman (1990, Experiment 2) reported a strong effect of changing the perceived meaning of a word between study and test. Their design was similar to that of Carroll and Kirsner (1982), except that single words were tested, all targets were homographs, and, similar to Smith et al. (1989), the intervals between repetitions were short (15 items). The context word biased a particular meaning of the target homograph. When the cue differed at test, but biased the same meaning, a smaller reduction in repetition priming was observed (in comparison with the same-cue condition) than when the context biased the alternative meaning. Thus, Masson and Freedman's (1990) data show that context can affect the meaning that is selected for encoding a repeated item and that reinstating a particular meaning does not depend on the repetition of a particular contexttarget pair.

The foregoing experiments suggest that repetition priming of predominantly data-driven tasks tends to be insensitive to changes in context, with the specific exception that repetition priming can be eliminated by altering the perceived meaning of a homograph. In part, the general context insensitivity may have resulted from the use of single cue words to manipulate context-we propose that a more satisfactory control can be obtained by the use of sentences. It has been shown that selective activation of the context-appropriate meaning of a homograph-that is, understanding cOUNT to mean "duke"-COUNT-occurs only if the preceding context sentence is sufficiently constraining. For example, counT would be constrained by "The vampire was disguised as a handsome counT"' but not by "The king kept losing track of the counT" (Simpson, 1981). By analogy, it seems likely that a single word context, such as that used by Masson and Freedman (1990), is less likely to impose a specific contextual interpretation that delimits the meaning of a stimulus.

Moreover, even for nonhomographs, numerous experiments have shown that the specific sense ${ }^{1}$ carried by a word in a sentence context is more restricted than is the interpretation of the same word presented alone or with a single word cue (see, e.g., Anderson \& Ortony, 1975; Glucksberg, Kreuz, \& Rho, 1986; O'Seaghdha, 1989; Sanocki \& Oden, 1984; Schuberth, Spoehr, \& Lane, 1981; Tabossi, 1988; Tabossi \& Johnson-Laird, 1980). To illustrate, consider one of Tabossi's (1988, Experiment 2) studies, which showed that lexical decisions were facilitated only when the target was related to a contextrelevant property of a preceding sentence-final noun. For example, responses to FAT would be facilitated by the preceding sentence, "To follow her diet, the woman eliminated the use of BUTTER." This facilitation must have reflected the specific linguistic context provided by the sentence, as opposed to some more general semantic priming between BUTTER and FAT, because facilitation of FAT was absent in sentences such as, "To soften it, the woman heated the piece of BUTTER," which focused on properties of BUTTER not relevant to the target.

If one concludes that selection of a specific nuance or sense of a word demands the presence of a strong, sentential context, it follows that the failure to find any systematic context effects in implicit memory (see, e.g., 
Smith et al., 1989) may well be due to the use of singleword context cues. Lewandowsky, Kirsner, and Bainbridge (1989) reported data supportive of this position. Subjects made a lexical decision about a homographic target that followed a sentence frame that biased one specific meaning. Similar to Masson and Freedman's (1990) finding, Lewandowsky et al. (1989) found intact repetition priming following a change in context but not meaning, but only when using the subordinate meaning of the homograph at test. When the dominant meaning was presented at test, a change in context reduced repetition priming. For example, responses to BANK were not primed when the preceding context sentence changed from "The teller worked at the ..." in Block 1 to "The robber held up the BANK" in Block 2. At the same time, a concomitant change in context failed to affect repetition priming when the subordinate (river-BANK) meaning of a homograph was biased at both study and test.

Lewandowsky et al. (1989) proposed that dominance maps into the number of nuances possessed by one meaning of a homograph, which in turn maps into the number of separate representations. It follows that more representations exist for the dominant than for the subordinate meaning, thus decreasing the likelihood for the former that repetition of a word in a different context (but biasing the same meaning) would access the same representation. According to this view, priming is a consequence of accessing the same, context-specific, representation of the word at both study and test. We refer to this as a sensespecific modification of the activation view (e.g., Graf \& Mandler, 1984; Morton, 1979), which holds that presentation of a stimulus "activates" the corresponding preexisting representation in lexical memory. Subsequent processing of an activated representation is facilitated, even in the absence of awareness of a prior study episode. Although the simple activation view has been found wanting in the face of numerous contrary results (for a discussion, see Schacter, 1987), its sense-specific variant is ideally suited for the straightforward presentation of our predictions and results. Comparisons with other theoretical accounts will be offered in the General Discussion.

Because the majority of nonhomographic words are, to varying degrees, polysemous (i.e., having a number of distinct nuances or senses), our modified activation view predicts that the extent to which a change in context affects repetition priming depends on the number of unique representations of the target. Items with a large number of senses should show reduced or absent priming when context is changed between presentations, whereas priming of words with fewer senses should not suffer in the same way. The following experiments were designed to explore this prediction.

\section{EXPERIMENT 1}

\section{Overview}

Subjects participated in two blocks of lexical decision trials, in which a sentence frame provided the context for each lexical decision and determined the likely interpretation given to a nonhomographic, but polysemous, item. Emphasis was on Block 2, in which repetition priming was compared across two context conditions-one in which the same sense as in Block 1 was biased and one in which a different sense was biased.

A given trial in either block would begin with presentation of a sentence that was missing the final word but that could either be completed with a single noun ("The man kicked the machine after it returned his ...") or, on a few filler trials, had no grammatical completion ("Kicked the man machine the it after returned his ..." "). To ensure careful reading of the context frame, the subjects had to decide whether, with a meaningful completion, the sentence would be grammatical or ungrammatical. Immediately following this decision, the sentence disappeared, and a single item was presented for lexical decision. When the item was a word, it would always form a meaningful completion for a preceding grammatical sentence (e.g., TOKEN), otherwise it was a pronounceable, but misspelled, version of the corresponding word (TOCIN).

Across blocks, some of the lexical decision targets were repeated. The primary manipulation consisted of the change, if any, in the contextual frame between first and second presentation. In the same-context $(S)$ condition, the same sentence frame was used on both occasions. In the different-context $(D)$ condition, a different sentence was used in Block 2 . The control condition $(N)$ consisted of words and context sentences that were presented for the first time in Block 2.

\section{Method}

Subjects. Twenty-four University of Oklahoma undergraduates participated for course credit.

Materials. One hundred ninety-two stimulus words-96 few-sense and 96 many-sense words - were selected from Webster's New World Dictionary (1979) according to the following constraints: Each word was a noun having only one entry (thereby disqualifying homographs), and the number of senses was defined as the number of distinct, numbered senses specified for the word. Few-sense words had a mean of 1.2 dictionary senses; many-sense words had a mean of 5.4 senses $[t(190)=23.79]$. All words had a frequency of less than $30 \mathrm{wpm}$ (as determined by Kučera \& Francis, 1967), with a mean of 3.2 wpm for few-sense items (mean length 6.9 letters) and a mean of $8.6 \mathrm{wpm}$ for many-sense items (mean length 5.82).

Data from a pilot experiment indicated that more important than the number of dictionary senses was the number of working, or available, senses (Gernsbacher, 1984). For most people, the average number of working senses of a word is at most two or three (Jorgensen, 1990). Therefore, 20 University of Oklahoma undergraduates rated the initial pool of words to determine the number of available senses. The subjects were tested in groups of 5 and were presented with booklets of 96 words (48 few-sense and 48 many-sense words); they were instructed to write down as many senses as they could for each item and to write "not known" if they did not know the word. These ratings were analyzed using an average number of meanings metric (Millis \& Button, 1989).

Forty-eight many-sense items and 48 few-sense items, with an average of 1.86 and 1.2 available senses, respectively, were selected from the initial pool to maximize the difference between the two sets of items. This final selection of 96 items had a frequency of less than 30 wpm (Kučera \& Francis, 1967), with a mean of 
2.1 wpm for few-sense iterns (mean length 6.75 letters) and a mean of $6.65 \mathrm{wpm}$ for many-sense items (mean length 5.62).

Context sentences for the many-sense items were designed so that perceived meaning was changed across presentations. For example, one context frame for TOKEN was "The man kicked the machine after it returned his ..."; the alternative sentence was "The young widow kept her husband's hair as a ..." Sentence frames for the few-sense items necessarily maintained a single sense across presentations. For example, the few-sense item Marina followed either "The thief stole the small vessel from its ..." or "The hurricane destroyed most of the boats in the ...",

Design and Procedure. The first block consisted of 222 trials, 148 involving words and 74 involving pseudowords. Twenty of the word trials and 14 of the pseudoword trials were preceded by ungrammatical sentences. In Block 2, the number of trials was 240 , 160 involving words and the remaining 80 involving pseudowords. Orthogonal to lexical status, one in every five sentences (32 and 16 for words and pseudowords, respectively) was ungrammatical.

The critical items ( 32 in Block 1 and 48 in Block 2) were divided evenly into many and few senses by pairing the appropriate word with the context sentence. The sense variable was fully crossed with the three context conditions $(S, D$, and $N$ ) to yield 16 replications per subject cell. Each target word was used equally often in each of the three experimental conditions across subjects. For a given target word in the $D$ condition, context was manipulated for half of the subjects by using one of the two possible sentences in Block 1 and by using the alternative sentence in Block 2 . This assignment was reversed for the remaining subjects.

The remaining sentences and lexical decision items in both blocks were randomly sampled from the pool of fillers for each subject. In addition, a random 64 of the grammatical filler sentences involving words in Block 1 were repeated in Block 2, except that half of them were now followed by the alternative, novel, completion word and the remaining half by a pseudoword. Thus, repetition of a sentence was not predictive of the target.

\section{Results and Discussion}

The analyses focused on lexical decisions in the experimental conditions in Block 2, using percent errors and latency of correct responses. Both measures were conditionalized on a correct yes response to the preceding grammaticality judgment. A lexical decision following an incorrect grammaticality judgment (a false no) did not contribute to the data for that particular condition. In addition, to eliminate the undue influence of extremely slow responses and premature keypresses, a trial was discarded if the latency of either judgment was shorter than $100 \mathrm{msec}$ or longer than $5 \mathrm{sec}$. The proportion of critical observations thus trimmed was under $1 \%$ in all experiments. Individual latencies were logarithmically transformed, averaged across replications within each condition for a given subject, and antilog transformed to yield one score (in millisecond units) per condition per subject for the analyses of variance (ANOVAs). All ANOVAs were completely within subjects and tested each effect against its interaction with the subject component as error term, at a significance level of .05.

Reaction times and error rates are shown in Table 1. The subjects made more errors responding to the fewsense words than to the many-sense words $[F(1,23)=$ $\left.57.22, M S_{\mathrm{e}}=.0082\right]$, but no other effect of accuracy was
Table 1

Response Latencies for Correct Lexical Decisions (in Milliseconds) in Block 2 in Experiment 1

\begin{tabular}{lcccc}
\hline & \multicolumn{4}{c}{ Number of Senses of Target } \\
\cline { 2 - 5 } \multicolumn{1}{c}{ Condition } & Many & Error Rates & Few & Error Rates \\
\hline Same context & 653 & .05 & 815 & .14 \\
Different context & 710 & .03 & 801 & .13 \\
Control & 755 & .05 & 936 & .20 \\
\hline
\end{tabular}

significant $\left[F(2,46)=2.12, M S_{\mathrm{e}}=.0109\right.$, and $F(2,46)=$ $1.44, M S_{\mathrm{e}}=.0092$, for context and the interaction, respectively].

Analysis of reaction times showed an effect of sense $\left[F(1,23)=93.28, M S_{\mathrm{e}}=8,097\right]$, an effect of context $\left[F(2,46)=27.26, M S_{\mathrm{e}}=6,176\right]$, and most important, an interaction between the two $\left[F(2,46)=4.00, M S_{e}=\right.$ $6,774]$. The $L S D$ of $48 \mathrm{msec}$ indicated that repetition priming was unaffected by a change in context for the few-sense condition but was significantly reduced in the many-sense condition. Thus, the latency data followed the pattern expected from the modified activation view: When the perceived sense of a word was changed across presentationsprovided that more than one sense was actually availablerepetition priming was greatly reduced. If the change in context did not also change perceived sense, repetition priming remained intact.

Interpretation of these results must take into account a potential difficulty associated with the use of sentence frames. While performing the grammaticality judgmenteven on a novel sentence-subjects may generate predictions concerning the target item. Thus, an alternative account of the results might hold that predictability of the targets differed in systematic ways between sense conditions. Specifically, if the few-sense item, DIME, was more predictable from the preceding sentence frame than, say, the many-sense item, TOKEN, was from its context, then a reduced context effect could be expected for DIME because it was predictable in Block 2 , even in a novel context ( $D$ condition). To assess the extent of this potential difficulty, we asked two additional groups of 16 subjects to complete the sentence frames used in Experiment 1 with the first word that came to mind. Each subject received a booklet of 96 randomly ordered sentence frames comprising one sentence for each of the $\mathbf{4 8}$ many-sense and 48 few-sense items, with the other sentence presented to the other group of subjects. Sentence frames used in the many-sense conditions were completed more often with the critical items than were their few-sense counterparts. Averaging across both groups of subjects, mean completion rates were 4.85 (out of a possible 48) and 1.03 (out of 48) for the many-sense and the few-sense items, respectively $\left[F(1,30)=121.60, M S_{\mathrm{e}}=1.91\right]$. Although the direction of this difference (many-sense completions more predictable than few-sense completions) is counter to that which would predict our results, Experiment 2 was de- 
signed to replicate the differential context effect using stimuli with the desired difference in number of available senses but with equal predictability of sentence frames.

\section{EXPERIMENT 2}

\section{Method}

Selection of stimuli. A preliminary pool of 193 nonhomographic words was created by three University of Oklahoma undergraduate judges. These items were within the 10-30-wpm frequency range and were presumed, by the judges, to have either a single sense or three or more senses. The subsequent norming of these words was performed by two groups of 18 undergraduates. Each subject was presented with a booklet containing either 96 or 97 words (randomly assigned) and was asked to write down as many senses as possible for each item, within $45 \mathrm{sec}$.

The final selection of experimental items satisfied the following criteria: At least one sense was known to all subjects; for the singlesense items, no subject reported more than one sense; and for the many-sense items, at least $60 \%$ of the subjects reported two or more meanings. Even with these liberal criteria, we were only able to form a pool of $30 \mathrm{critical}$ items ( 15 single-sense and 15 many-sense), allowing only 5 items per subject cell. Mean frequency of words was $10.3 \mathrm{wpm}$ for single-sense items (mean length 6.1 letters), compared with $11.9 \mathrm{wpm}$ for many-sense items (mean length 5.27).

Each item was associated with two context sentences. As in Experiment 1 , the sentences in the many-sense condition were used to bias two different senses of an item, whereas in the single-sense condition, by definition, sentences could not change sense. For example, the many-sense item, TICKET, followed "The policeman pulled him over and gave him a ..." and "At the door to the cinema she collected a ..." These sentence frames were given to two additional groups of 16 subjects to assess predictability of targets. Similar to Experiment 1, each subject received a booklet containing 30 randomly ordered sentence frames. A given subject saw only one member of the pair of sentences used for an item, with the other sentence presented to a subject in the other group. Analyses of the subjects' completion rates showed no difference between the manysense (3.12 out of a possible 15 ) and single-sense (3.22 out of 15 ) conditions $\left[F(1,30)<1, M S_{\varepsilon}=1.61\right]$. Comparison across groups of subjects showed that both members of a sentence pair were equally predictive of the target: Completion rates were 3.44 and 2.93 (out of 15) for the two groups $\left[F(1,30)=1.91, M S_{\mathrm{e}}=2.09\right]$, with no interaction between sense and group $\left[F(1,30)<1, M S_{\mathrm{c}}=1.61\right]$. Thus, there was no evidence to suggest that sentence frames differed in predictability between levels of the sense variable or between members of the context pairs.

Subjects and Procedure. Forty University of Oklahoma undergraduates participated in this experiment. Except for the smaller number of critical items, and a correspondingly reduced total number of trials per block (80), the procedure was identical to that used in Experiment 1

\section{Results and Discussion}

Reaction times and error rates are presented in Table 2. An analysis of error rates showed an effect of sense and an effect of context but no interaction between the two $\left[F(1,39)=7.5, M S_{\mathrm{e}}=.0165, F(2,78)=4.15, M S_{\mathrm{e}}=\right.$ .0147 , and $F(2,78)<1$ for sense, context, and the interaction, respectively].

Owing to the small number of replications per subject cell, latency analyses used median reaction times (which are less sensitive to outlying observations). An ANOVA revealed that response latencies did not differ between the two sense conditions $(681 \mathrm{msec}$ and $660 \mathrm{msec}$ for the
Table 2

Response Latencies for Correct Lexical Decisions (in Milliseconds) in Block 2 in Experiment 2

\begin{tabular}{lcccc}
\hline & \multicolumn{4}{c}{ Number of Senses of Target } \\
\cline { 2 - 5 } \multicolumn{1}{c}{ Condition } & Many & Error Rates & Single & Error Rates \\
\hline Same context & 618 & .06 & 631 & .03 \\
Different context & 680 & .13 & 649 & .06 \\
Control & 682 & .11 & 763 & .07 \\
\hline
\end{tabular}

single-sense and many-sense conditions, respectively $\left[F(1,39)=1.53, M S_{\mathrm{e}}=17,987\right]$. On the other hand, both the context effect and the interaction between sense and context were significant $\left[F(2,78)=13.96, M S_{\mathrm{e}}=14,047\right.$, and $F(2,78)=3.07, M S_{e}=20,800$, respectively].

Subsequent analysis of that interaction, using the $L S D$ of $63 \mathrm{msec}$, confirmed that repetition priming was not affected by a change in context for items with a single sense. In contrast, items with many available senses were affected by a change in context, with no repetition priming in the different-context condition. To further illustrate this pattern, consider the relative priming values (Kirsner \& Dunn, 1985) for the two sense conditions. Relative priming (RP) values provide a measure of the transfer of priming and are calculated by forming a ratio between the facilitation found for the $D$ condition and the facilitation for the $S$ condition: $\mathrm{RP}=(D-N) /(S-N)$.

The RP values were .86 and .03 for the single- and many-sense conditions, respectively, indicating that a change in context reduced repetition priming by only $14 \%$ for words with a single sense, but by $97 \%$ for words with many senses.

\section{EXPERIMENT 3}

The two preceding experiments support the view that the repetition of perceived sense is an integral component of priming and that if a change in context changes the perceived sense, repetition priming is eliminated even with nonhomographic items. We predicted these results with respect to the activation of a meaning-specific representation of the target item. However, an alternative explanation would hold that the subjects' performance was a consequence of some form of explicit association between the context frame and the target. Because the subjects made the grammaticality judgments without knowledge of the sentence-final item, it is possible that upon appearance of the lexical decision target, the subjects would confirm their preceding grammaticality decision by verifying that the target meaningfully completed the sentence frame. Conceivably, this process might engender the formation of an explicit association between the target and its context frame. This association, in turn, would then be reinstated when the same context frame reappeared in Block 2, thus giving an undue advantage to same-context targets.

Although it is unclear how the association explanation would account for the striking differences between many- 
Table 3

Response Latencies for Correct Lexical Decisions (in Milliseconds) in Block 2 in Experiment 3

\begin{tabular}{lcccc}
\hline & \multicolumn{4}{c}{ Number of Senses of Target } \\
\cline { 2 - 5 } \multicolumn{1}{c}{ Condition } & Many & Error Rates & Single & Error Rates \\
\hline Same context & 648 & .02 & 699 & .03 \\
Different context & 713 & .04 & 693 & .04 \\
Control & 732 & .02 & 767 & .07 \\
\hline
\end{tabular}

and few-sense items in Experiments 1 and 2, the next study was designed to reduce the demand characteristics that might engender the formation of explicit associations between context and target. Thus, the subjects in Experiment 3 were required to read the context sentences aloud without making a decision about their grammaticality.

\section{Method}

Forty-nine University of Oklahoma undergraduates participated for course credit. The subjects in this experiment were required to read context sentences aloud but were not required to make a grammaticality decision. Therefore, we no longer used filler items with ungrammatical context sentences. Otherwise, the stimuli and procedures were identical to those used in Experiment 2.

\section{Results and Discussion}

Reaction times and error rates are shown in Table 3. An analysis of the error rates showed an effect of sense $\left[F(1,48)=5.96, M S_{\mathrm{e}}=0.0074\right]$ but no effect of condition, and no interaction between the two $[F(2,96)=1.27$, $M S_{\mathrm{e}}=0.0068$, and $F(2,96)=2.40, M S_{\mathrm{e}}=0.0048$, respectively].

Analyses of reaction times showed an effect of both sense and condition $\left[F(2,48)=3.35, M S_{\mathrm{e}}=10,771\right.$, and $F(2,96)=21.10, M S_{\mathrm{e}}=6,769$, respectively], and an interaction between the two $\left[F(2,96)=3.82, M S_{\mathrm{e}}=\right.$ $8,818]$. Subsequent analysis of the interaction using the $L S D$ of $38 \mathrm{msec}$ showed that repetition priming was eliminated when context was changed for the many-sense items but that repetition priming was maintained across a change in context for the single-sense items. The results confirm that the outcome of Experiments 1 and 2 was probably not caused by an explicit "backward" association between the context sentence and the target.

\section{GENERAL DISCUSSION}

\section{Summary}

We reported three experiments that clarified the role of context in repetition priming of lexical decisions. The results of all three experiments support the interpretation that priming depends not only on repetition of the same physical word form but also on the repetition of the same context-selected sense. When sense was changed, priming was eliminated, whereas a context change that did not also change perceived sense was inconsequential. The data from these experiments support previous findings (Lewandowsky et al., 1989; Masson \& Freedman, 1990) that demonstrated the importance of encoded meaning in repetition priming and, therefore, implicit memory. Thus, any view of implicit memory must acknowledge the role of context and context-selection of meaning.

\section{Sense-Specific Activation}

As stated at the outset, our working assumption was that the number of senses of a word is reflected in the number of its internal representations and that priming occurs if the same representation is reactivated at a later point. This sense-specific activation mechanism not only explains the outcome of our experiments but also sheds light on some diverse previous results.

Presentation in context. In our view, each time a word is encountered, its meaning is determined by its context, and the representation corresponding to that sense is activated. This specific and narrow interpretation given to a word is evident on later implicit tests of memory: If a different context is present, and there is a large number of alternative representations for a word (dominant meaning of homographs or polysemous words), repetition priming is likely to be absent. If there are fewer representations (subordinate meaning, or words with few senses), priming may persist in a different context-unless perceived sense is also changed-because the probability of accessing the same representation again is higher.

Context-free presentation. Words are often encountered, and understood, without any recourse to a specific context. In these situations, words have a preferred customary meaning (Allan, 1981). For example, the customary meaning of LAMB would refer to the meat, and only when context is present would it also refer to the pelt, the head, or still other nuances. Because the customary meaning will, on average, be activated each time a word is presented in isolation, repetition of isolated words facilitates processing.

Now consider the effects of removing context between study and text. When study words are part of continuous text and are later presented in isolation for perceptual identification (see, e.g., Levy \& Kirsner, 1989), lexical decisions (see, e.g., Oliphant, 1983), or fragment completion (MacLeod, 1989), repetition priming is absent or, at best, minimal. In our view, a specific, context-selected meaning was encoded at study, whereas the isolated presentation at test favored encoding of the less specific customary meaning. Because these two meanings frequently do not overlap, little or no priming is observed. Interestingly, in MacLeod's study, considerably more priming accrued to words that, although also "part" of the study text, were not constrained by context. The subjects had to identify and cross out words that did not fit meaningfully into a passage of text, and these words, unbound by surrounding context, were primed more than were words that were meaningfully integrated into the passage. In our view, the lack of integrative context permitted selection of the customary sense on both occasions, thus leading to repetition priming in the same way that single items are facilitated across blocks. 
A similar explanation applies to the studies described earlier that used single words to constrain context. In the experiment by Smith et al. (1989), pairs of words, either intact or repaired across repetitions, showed equivalent repetition priming. Because the single context words were not semantically related to the targets, they were unlikely to have constrained interpretation in any way. Thus, on average, the same customary meaning of a target was selected at both study and test, and as a consequence, Smith et al. found identical priming for words presented in the same or different contexts.

\section{A Processing Explanation}

We have shown that numerous context effects can be handled with respect to a modified sense-specific activation view. However, because many previous empirical comparisons have favored alternative theoretical accounts, the possibility exists that our data might be better explained in some other way, or at least within a more encompassing framework that subsumes sense-specific activation.

Most prominent among competing theoretical accounts is the processing view of implicit memory (Graf \& Ryan, 1990; Kolers \& Roediger, 1984; Roediger \& Blaxton, 1987; Roediger, Srinivas, \& Weldon, 1989), which holds that implicit memory is the consequence of the test reinstating the same cognitive operations that were engaged during encoding. Thus, if a given set of operations is used at study, implicit memory is observed if these, or similar, operations are reinstated at test. The processing view has an impressive track record, accurately predicting both dissociations and associations between various implicit memory tasks (Roediger et al., 1989).

Despite its emphasis on reinstatement, the original processing view may have difficulty accounting for context effects: Little reference is made to the linguistic details of a study episode, and cognitive processes are broadly classified as either data-driven or conceptually driven. If implicit memory is the result of reinstating the cognitive processes used at encoding, and if it is their global nature (whether they are conceptually or data-driven) that principally determines the extent of reinstatement, then the processes applied to, say, TOKEN at its first encoding should be reinstated at its second occurrence, regardless of perceived sense.

To accommodate context effects, then, proponents of the processing view would need to argue that context is intrinsic to the processes to be reinstated. Indeed, Masson and MacLeod (1992) presented one such contextsensitive modification of the processing view. Their proposal distinguishes between initial encoding processes, which develop a context-specific interpretation of the stimulus, and their subsequent elaboration. Under this proposal, explicit memory tasks rely primarily on the latter, elaborative, processes, whereas implicit memory tasks rely more on the initial encoding stages. Unlike the original processing view, Masson and MacLeod's proposal acknowledges that both data-driven and conceptually driven processes contribute to initial encoding. Priming occurs when a set of initial encoding processes is reinstated at test. Because context, like other conceptual variables, may enter into these processes, priming is reduced when context is changed.

Because Masson and MacLeod (1992) specifically include the encoded nuances of a word's meaning to be among the processes benefiting from later reinstatement (p. 147), their view is nearly isomorphic to the reasoning offered here for many-sense items. However, we argue that Masson and MacLeod's view cannot also account for the absence of a context effect for targets with few senses or only a single sense. Although these words necessarily lead to encoding of the same nuance on both occasions, Masson and MacLeod explicitly state that memorial representations depend on "processing knowledge beyond the boundaries of the target item" (p. 164). Hence, reinstatement of that representation should suffer if context is changed, even if the target has only one sense. Our data show otherwise.

\section{Conclusion}

We proposed that at least some manifestations of implicit memory arise from the activation of context-selected meanings of a word. Repetition priming, in this view, depends on encoding of the same sense at study and test. If a change in context also alters perceived sense, repetition priming is reduced or eliminated. A concomitant context change that does not also change perceived sense remains inconsequential. The overall pattern of results appeared best summarized by a modified activation view, in preference to a variant of the processing view.

\section{REFERENCES}

ALLAN, K. (1981). Interpreting from context. Lingua, 53, 151-173. ALLEN, S. W., \& JACOBY, L. L. (1990). Reinstating study context produces unconscious influences of memory. Memory \& Cognition, 18, 270-278.

ANDERSON, R. C., \& ORTONY, A. (1975). On putting apples into bottles-A problem of polysemy. Cognitive Psychology, 7, 167-180.

BLAXTON, T. A. (1989). Investigating dissociations among memory measures: Support for a transfer-appropriate processing framework. Journal of Experimental Psychology: Learning, Memory, \& Cognition, 15, 657-668.

Carroll, M., \& Kirsner, K. (1982). Context and repetition effects in lexical decision and recognition memory. Journal of Verbal Learning \& Verbal Behavior, 21, 55-69.

GernsBacher, M. A. (1984). Resolving 20 years of inconsistent interactions between lexical familiarity and orthography, concreteness, and polysemy. Journal of Experimental Psychology: General, 113, 256-281.

Glucksberg, S., Kreuz, R. J., \& Rho, S. H. (1986). Context can constrain lexical access: Implications for models of language comprehension. Journal of Experimental Psychology: Learning, Memory, \& Cognition, 12, 323-335.

Graf, P., \& Mandler, G. (1984). Activation makes words more accessible, but not necessarily more retrievable. Journal of Verbal Learming \& Verbal Behavior, 23, 553-568.

GRaF, P., \& RYAN, L. (1990). Transfer-appropriate processing for implicit and explicit memory. Joumal of Experimental Psychology: Learning, Memory, \& Cognition, 16, 978-992.

Graf, P., \& SCHACTER, D. L. (1985). Implicit and explicit memory 
for new associations in normal and amnesic subjects. Journal of Ex perimental Psychology: Learning, Memory, \& Cognition, 11, 501-518.

GraF, P., \& SChacter, D. L. (1987). Selective effects of interference on implicit and explicit memory for new associations. Journal of Experimental Psychology: Learning, Memory, \& Cognition, 13, 45-53.

GRAF, P., \& SCHACTER, D. L. (1989). Unitization and grouping mediate dissociations in memory for new associations. Journal of $E x$ perimental Psychology: Learning, Memory, \& Cognition, 15, 930-940.

Hamann, S. B. (1990). Level-of-processing effects in conceptually driven implicit tasks. Journal of Experimental Psychology: Learning Memory, \& Cognition, 16, 970-977.

$\mathrm{J}_{\mathrm{ACOBY}}$, L. L. (1983). Remembering the data: Analyzing interactive processes in reading. Joumal of Verbal Learning \& Verbal Behavior, 22, 485-508.

JACOBY, L. L., \& DALLAS, M. (1981). On the relationship between autobiographical memory and perceptual learning. Joumal of Experimental Psychology: General, 110, 306-340.

JORGENSEN, J. C. (1990). The psychological reality of word senses. Journal of Psycholinguistic Research, 19, 167-190.

Kirsner, K., \& DUNN, J. C. (1985). The perceptual record: A common factor in repetition priming and attribute retention. In M. I. Posner \& O. S. M. Marin (Eds.), Attention and performance XI (pp. 547565). Hillsdale, NJ: Erlbaum.

Kolers, P. A., \& RoEdiger, H. L., III (1984). Procedures of mind. Journal of Verbal Learning \& Verbal Behavior, 23, 425-449.

KuČERA, H., \& FranCIs, W. N. (1967). Computational analysis of present-day American English. Providence, RI: Brown University Press.

LeVy, B. A., \& KirSNer, K. (1989). Reprocessing text: Indirect measures of word and message level processes. Joumal of Experimental Psychology: Learning, Memory, \& Cognition, 15, 407-417.

Lewandowsky, S., Kirsner, K., \& Bainbridge, J. V. (1989). Context effects in implicit memory: A sense-specific account. In S. Lewandowsky, J. C. Dunn, \& K. Kirsner (Eds.), Implicit memory: Theoretical issues (pp. 185-198). Hillsdale, NJ: Erlbaum.

MACLEOD, C. M. (1989). Word context during initial exposure influences degree of priming in word fragment completion. Journal of Experimental Psychology: Learning, Memory, \& Cognition, 15, 398-406.

Masson, M. E. J., \& Freedman, L. (1990). Fluent identification of repeated words. Joumal of Experimental Psychology: Learning, Memory, \& Cognition, 16, 355-373.

Masson, M. E. J., MacLeod, C. M. (1992). Re-enacting the route to interpretation: Enhanced perceptual identification without prior perception. Journal of Experimental Psychology: General, 121, 145-176.

Miluis, M. L., \& ButTon, S. B. (1989). The effect of polysemy on lexical decision time: Now you see it, now you don't. Memory \& $\mathrm{Cog}$ nition, 17, 141-147.

Monsell, S. (1985). Repetition and the lexicon. In A. W. Ellis (Ed.), Progress in the psychology of language (Vol. 2, pp. 147-195). Hillsdale, NJ: Erlbaum.

Morton, J. (1979). Word recognition. In J. Morton \& J. C. Marshall (Eds.), Psycholinguistics: 2. Structure and processes. Cambridge, MA: MIT Press.
Oliphant, G. W. (1983). Repetition and recency effects in word recognition. Australian Journal of Psychology, 35, 393-403.

O'Seaghdha, P. G. (1989). The dependence of lexical relatedness effects on syntactic connectedness. Journal of Experimental Psychology: Learning, Memory, \& Cognition, 15, 73-87.

RoEDIGER, H. L., III, \& BLAXTON, T. A. (1987). Effects of varying modality, surface features, and retention interval on priming in wordfragment completion. Memory \& Cognition, 15, 379-388.

Roediger, H. L., III, SRinivas, K., \& Weldon, M. S. (1989). Dissociations between implicit measures of retention. In $S$. Lewandowsky, J. C. Dunn, \& K. Kirsner (Eds.), Implicit memory: Theoretical issues (pp. 67-84). Hillsdale, NJ: Erlbaum.

SANOCKI, T., \& ODEN, G. C. (1984). Contextual validity and the effects of low-constraint sentence contexts on lexical decisions. Quarterly Journal of Experimental Psychology, 36A, 145-155.

Scarborough, D. L., Cortese, C., \& Scarborough, H. S. (1977). Frequency and repetition effects in lexical memory. Journal of $E \boldsymbol{x}$ perimental Psychology: Human Perception \& Performance, 3, 1-17.

SCHACTER, D. L. (1987). Implicit memory: History and current status. Journal of Experimental Psychology: Learning, Memory, \& Cognition, 13, 501-518.

Schuberth, R. E., Spoehr, K. T., \& Lane, D. M. (1981). Effects of stimulus and contextual information on the lexical decision process. Memory \& Cognition, 9, 68-77.

Simpson, G. B. (1981). Meaning dominance and semantic context in the processing of lexical ambiguity. Journal of Verbal Learning \& Verbal Behavior, 20, 120-136.

Smith, M. C., Macleod, C. M., Bain, J. D., \& Hoppe, R. B. (1989). Lexical decision as an indirect test of memory: Repetition priming and list wide priming as a function of type of encoding. Journal of Experimental Psychology: Learning, Memory, \& Cognition, 15, $1109-1118$.

TABOSSI, P. (1988). Effects of context on the immediate interpretation of unambiguous nouns. Journal of Experimental Psychology: Learning, Memory, \& Cognition, 14, 153-162.

TABosSI, P., \& Johnson-Laird, P. N. (1980). Linguistic context and the priming of semantic information. Quarterly Joumal of Experimental Psychology, 32, 595-603.

Tulving, E., Schacter, D. L., \& Stark, H. A. (1982). Priming effects in word-fragment completion are independent of recognition memory. Joumal of Experimental Psychology: Learning, Memory, \& Cognition, 8, 335-342.

Webster's new world dictionary of the American language (2nd ed.) (1979). Cleveland, OH: William Collins.

\section{NOTE}

1.We have chosen to differentiate between "meaning" and "sense", to maintain a distinction between homographs-words that do have distinct meanings-and polysemous words, which may be used with alternative senses without necessarily involving distinct meanings.

(Manuscript received July 19, 1991; revision accepted for publication March 22, 1993.) 